\title{
Time-temperature dependent fracture toughness of PMMA
}

\section{Part 2}

\author{
A. G. ATKINS, C. S. LEE, R. M. CADDELL \\ Department of Mechanical Engineering, University of Michigan, Ann Arbor, Michigan, USA
}

Some observations are made on the fractography of surfaces obtained by cracking "compact tension" profile testpieces of PMMA over a range of temperatures and crack speeds, both stably and unstably. To a first approximation, it was possible to group and "shift" (as in visco-elastic transformations) characteristic surface markings at various fracture toughness/temperature/crack velocity combinations, particularly in the range where a toughness-biased Ree-Eyring relationship described the experimental toughness data.

\section{Introduction}

In Part 1 [1], we investigated the toughness of PMMA over a range of crack speeds. In contrast to earlier toughness studies which used different specimens to obtain different crack speeds (and hence crack tip strain rates), the same type of edge crack "compact tension"
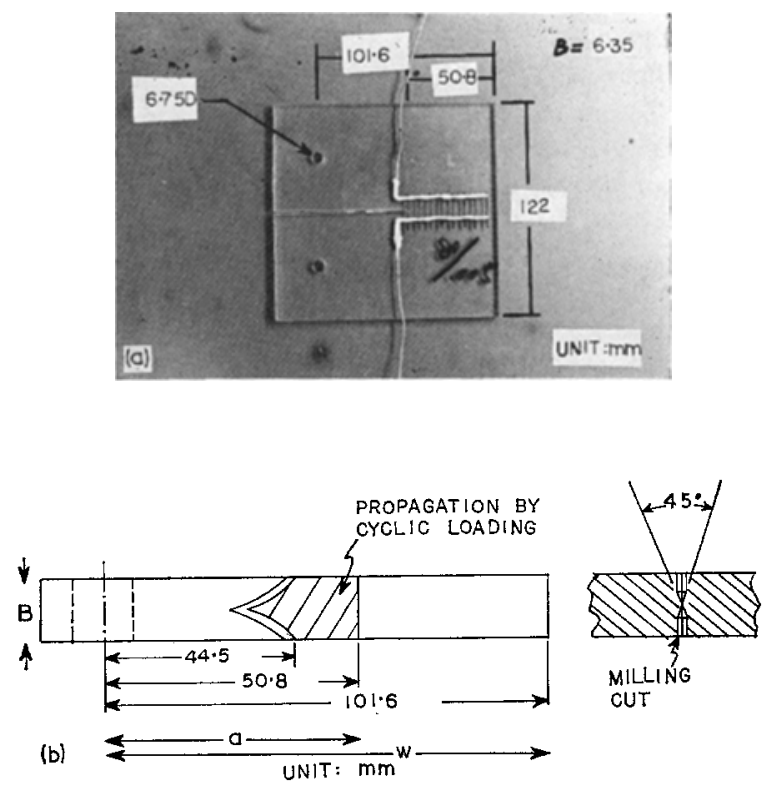

Figure 1 Geometry of testpiece used throughout the study. testpiece was used throughout (Fig. 1). This allowed slow and fast cracking, stable and unstable cracking, and changes in fractographic markings, to be observed in a controlled manner. In addition, Gurney's sector area technique [2] was used to measure the fracture toughness, $R$, which gave many values during crack propagation in one test. Conventional "fracture mechanics" measurements of the stress intensity factor, $K_{\mathbf{C}}$, usually give only one initiation value per specimen. Rate dependencies in $R$ can be observed in single tests since the crack velocity usually decreases during propagation in edge crack specimens.

This paper discusses the fracture surface markings at different combinations of fracture toughness, temperature and crack speed in PMMA. Stable and unstable features, wellknown in the fracture of glass, were observed. Conic markings help to identify factors affecting crack initiation in fast fracture, and the importance of void formation and crazing in these polymers is brought out. Surface markings characteristic of given toughness $(R)$, temperature $(T)$ and crack velocity $(\dot{a})$ combinations were found to correlate, particularly in the range where a "toughness-biased" Ree-Eyring relationship for $(R, T, \dot{a})$ applied [1]. It seems that, at least qualitatively, surface markings at different temperatures and crack speeds may be shifted to a "master-curve", analogous to general visco-elastic transforms. 


\section{General considerations}

Figs. 2 to 5 show the fracture surfaces of PMMA edge-crack toughness specimens at various temperatures and testing machine cross-head speeds. Light gold coatings were employed to provide better reflection from the otherwise transparent surfaces.

In general, experiments at high temperatures and low cross-head speeds gave stable cracks. The testing machine load $(P)$, cross-head displacement $(u)$ plots during stable propagation follow toughness loci that would be anticipated for the geometry of the edge-crack specimen used (Fig. 6a). Stable cracking occurred over a range of crack propagation velocities between $10^{-5}$ and $10^{-1} \mathrm{~m} \mathrm{sec}^{-1}$, and crack velocity depended much more on testing machine cross-head velocity than on the test temperature. The specimen geometry used in the tests was such that the cracks slowed down during the experiments, almost by one order of magnitude by the time the crack reached the back face of the testpiece.

With very low stable crack speeds $(\sim 10 \mu \mathrm{m}$ $\left.\mathrm{sec}^{-1}\right)$, the fracture surfaces are smooth and featureless to the naked eye. As the crack velocities increase, the fracture surfaces become rougher, with rib markings appearing along the direction of crack propagation. At low crack speeds, the ribs appear as numerous needle-like markings; they become coarser at high speeds. Certain temperatures caused rapid reductions in toughness for small changes in crack velocity [1]; this led to sudden changes in fracture patterns within small propagation distances in single specimens, as illustrated in Fig. 7.

Attempts to propagate cracks faster than about $100 \mathrm{~mm} \mathrm{sec}-1$ at room temperature produced unstable cracks, and the fracture surfaces reverted to a smooth flat appearance. To the naked eye this was again featureless. Unstable cracking was accompanied by sudden drops in load at constant cross-head displacement (Fig. 6b); since the "geometric stability factor" of the edge crack specimen favours stable crack propagation, the instabilities that are observed may be attributed to a sudden drop in the toughness parameter caused by an isothermal/adiabatic transformation $[1,3,4]$.

Sometimes crack arrest occurred at combina-
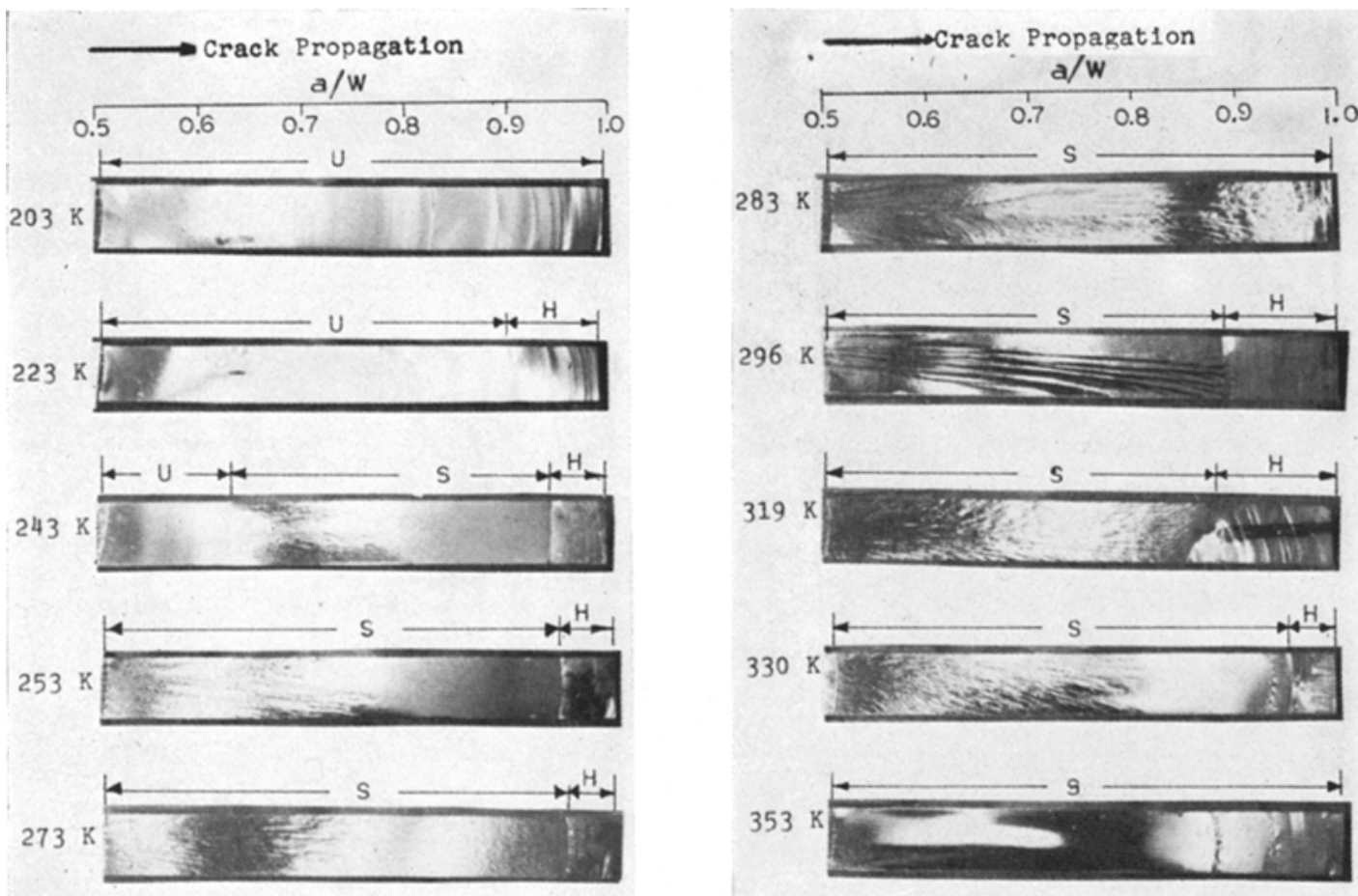

Figures 2 to $5 \mathrm{In}$ all these figures $\mathrm{S}$ indicates stable cracking, $\mathrm{U}$ unstable, and $\mathrm{H}$ broken by hand.

Figure 2 Fracture surfaces of PMMA at various temperatures broken with a cross-head speed of $8.33 \mu \mathrm{m} \mathrm{sec}-1$. 

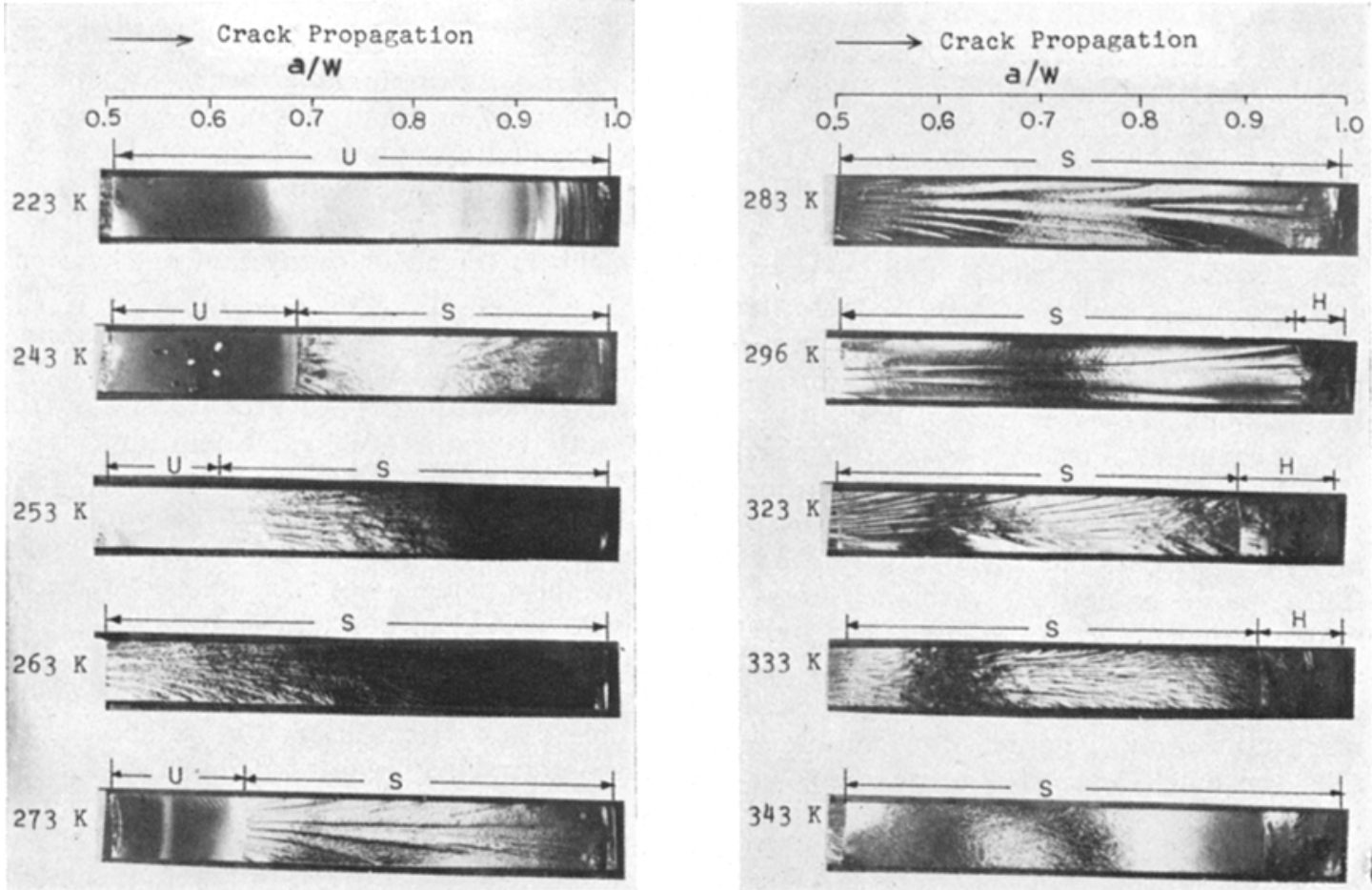

Figure 3 Fracture surfaces of PMMA at various temperatures broken with a cross-head speed of $83.3 \mu \mathrm{m} \mathrm{sec}^{-1}$.
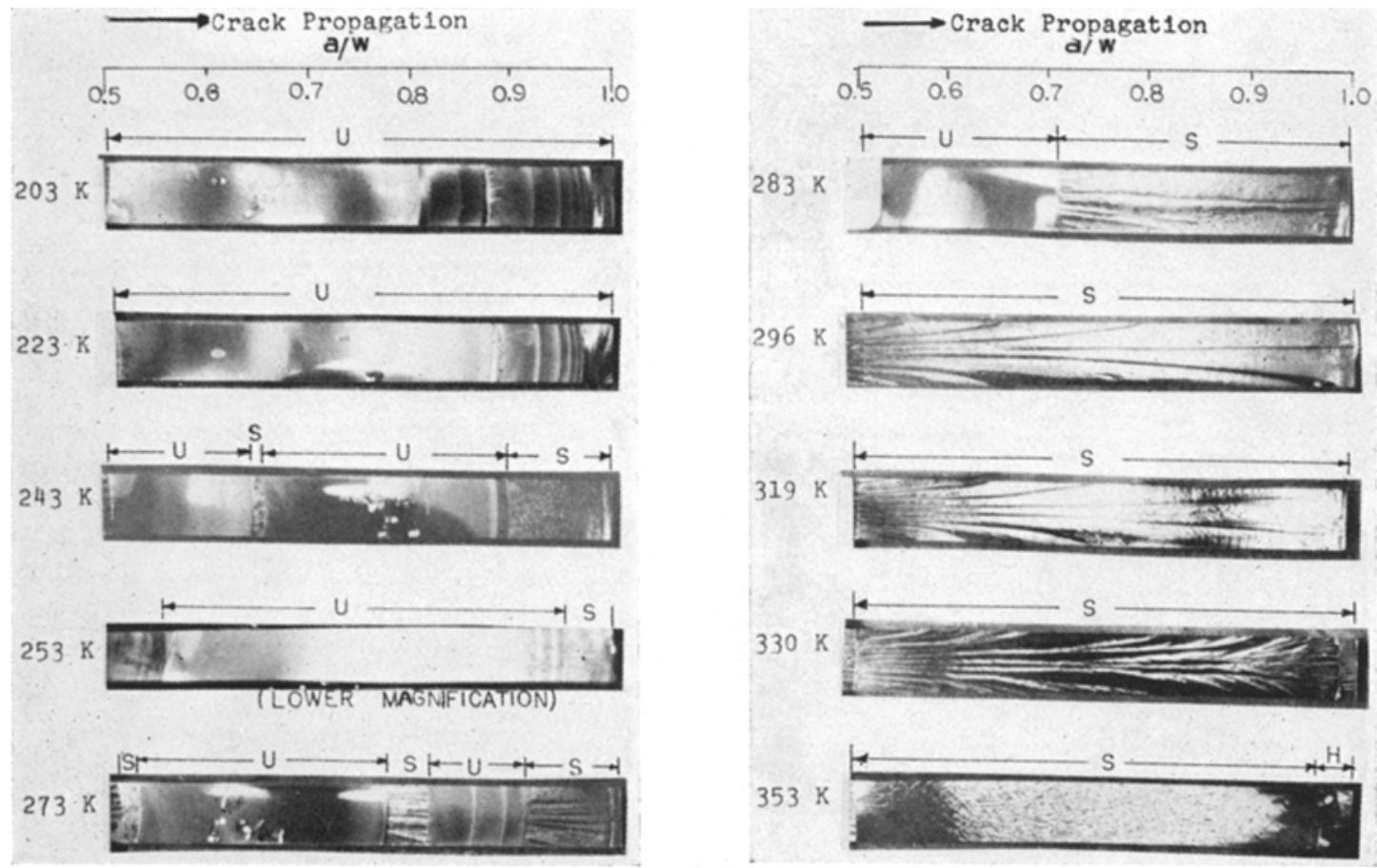

Figure 4 Fracture surfaces of PMMA at various temperatures broken with a cross-head speed of $833 \mu \mathrm{m} \mathrm{sec}-1$. 

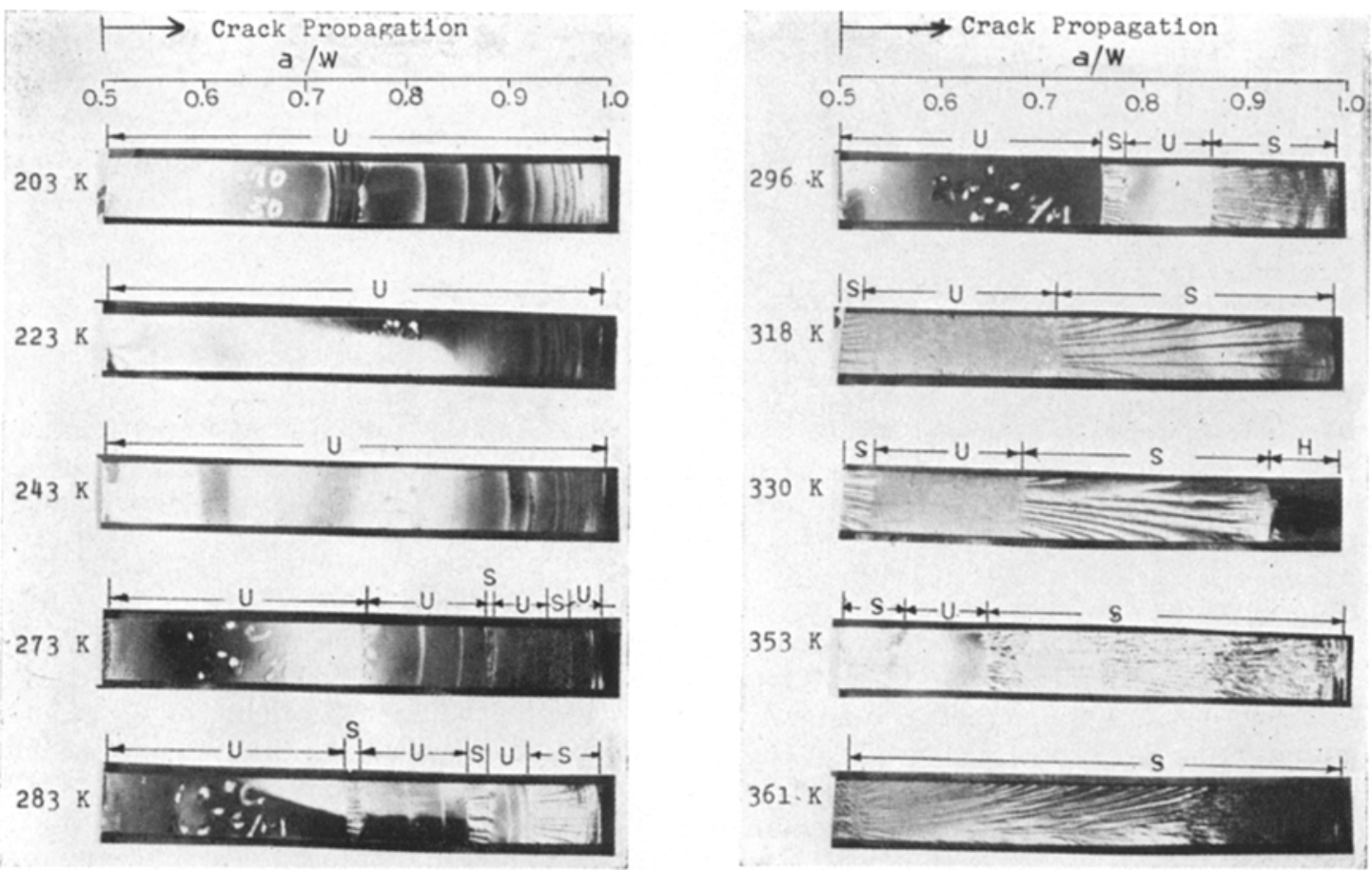

Figure 5 Fracture surfaces of PMMA at various temperatures broken with a cross-head speed of $8.33 \mathrm{~mm} \mathrm{sec}^{-1}$.
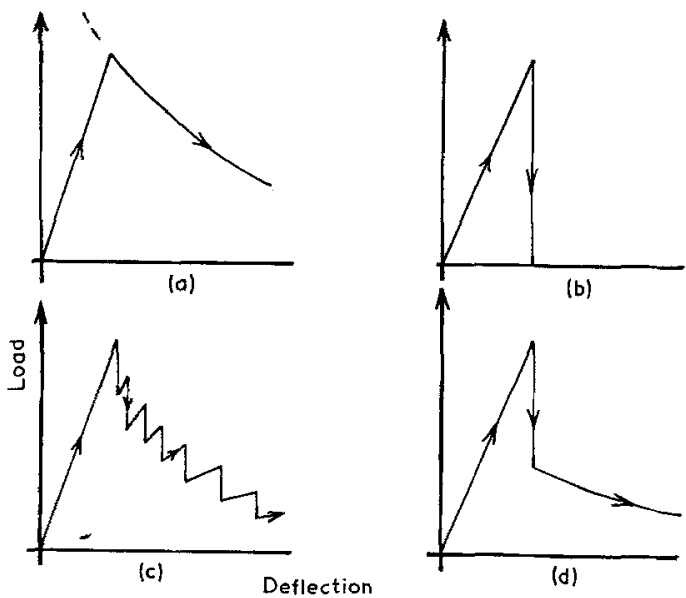

Figure 6 (a) Quasi-static $R$ locus for testpiece. (b) Sudden drop in load at constant cross-head displacement which accompanies unstable cracking. (c) Saw tooth load/ displacement response of crack arrest followed by increasing load for cracking. (d) Sudden unstable load drop followed by quasi-static $R$ locus.

tions of low temperatures and high cross-head speeds. Arrest appears as a distinct line across the fracture surface. Subsequent propagation after arrest was sometimes again unstable, followed by arrest and then another instability, etc. (which produced a sawtooth load-extension plot as in Fig. 6c), or sometimes it was stable and followed a quasi-static toughness locus (Fig. 6d).

\section{Flat fractures, conical markings and Wallner lines}

The two types of flat fractures, i.e. those generated during very slow stable cracking and those generated at the initially unstable crack speeds, can be distinguished under the microscope. The pairs of fracture surfaces shown in Fig. 8 have been obtained from both extremes of crack speed at the same temperature. When viewed through an optical microscope $(\times 70)$, the smooth surfaces of high speed fracture show conical markings. Those obtained in very slow cracking merely display some lines lying across the direction of propagation with some dimple marks scattered around the surface.

The conical markings on the fracture surfaces of unstable crack propagation are formed from the interaction between the primary crack front and secondary cracks generated ahead of the crack front. The particular form of conic produced depends on the value of $n$, the crack speed 

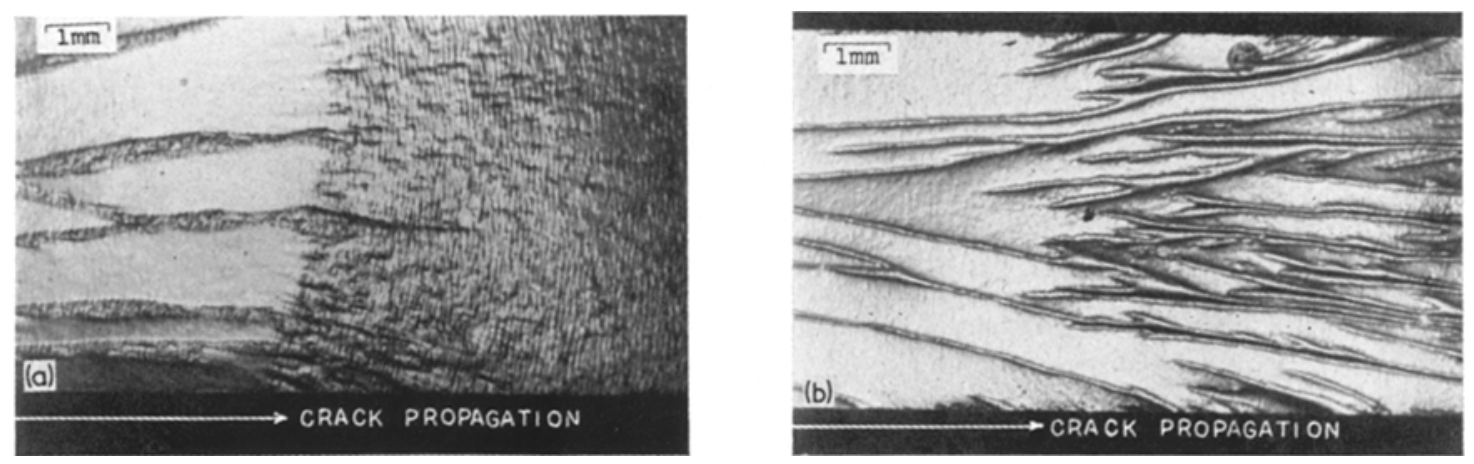

Figure 7 Abrupt change in PMMA fracture surface associated with the sudden change in fracture toughness at (a) $243 \mathrm{~K}$ and (b) $353 \mathrm{~K}$.

ratio, given by $n=v / V$, where $v$ is the velocity of the secondary crack and $V$ is the speed of the primary crack front. For $n<1$, an ellipse is produced, for $n=1$, a parabola, and for $n>1$, an hyperbola [see e.g. 5].

Fig. 9 shows various aspects of an unstable fracture surface resulting from pulling the specimen at $8.33 \mathrm{~mm} \mathrm{sec}-1$ at $193 \mathrm{~K}$. Fracture started at point $\mathrm{O}$, near the middle of the initial crack front where the highest stress is expected. Fig. $9 \mathrm{~b}$ shows the magnified view $(\times 150)$ of the fracture origin and Fig.9c shows some of the conical markings around the crack origin at greater magnification $(\times 2150)$. An elliptical marking is visible near the fracture origin in Fig. 9b. The location of the centre of the
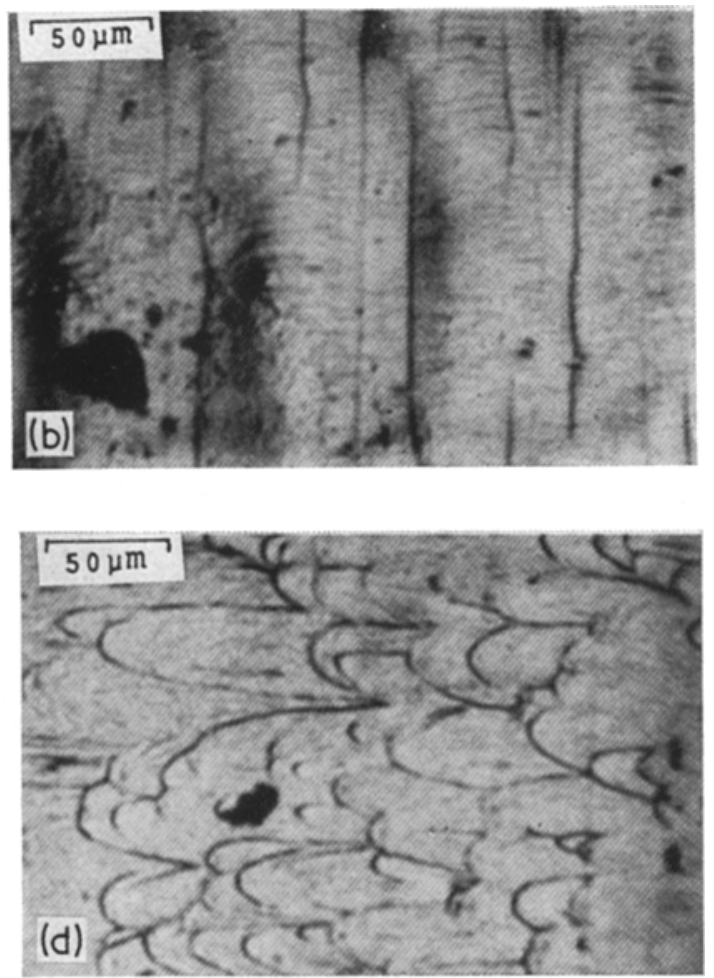

Figure 8 Differences in microscopic features of slow stable cracking at (a) $273 \mathrm{~K}$ and (b) $296 \mathrm{~K}$, and fast unstable fracture at (c) $273 \mathrm{~K}$ and (d) $296 \mathrm{~K}$. Crack propagation from left to right. 

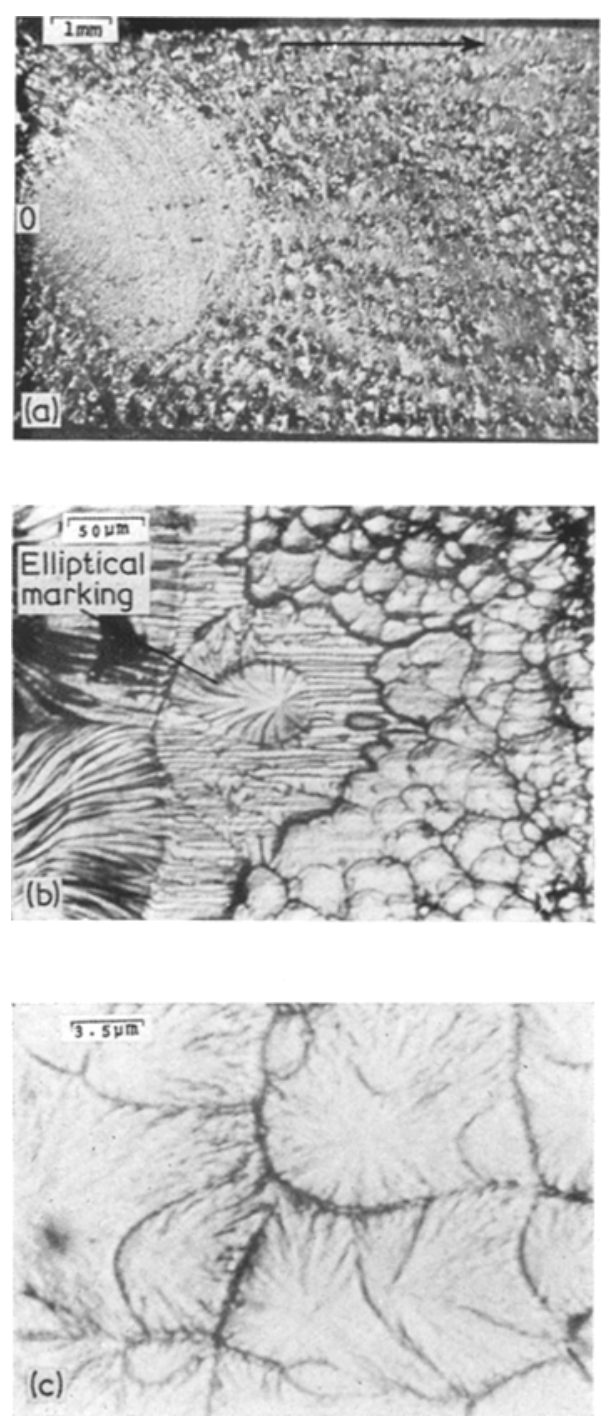

Figure 9 Features of an unstable crack surface (at various magnifications) at $193 \mathrm{~K}$ where cross-head velocity was $8.33 \mathrm{~mm} \mathrm{sec}^{-1}$.

ellipse is at $\left(x=x_{0} /\left(1-n^{2}\right), y=0\right)$ where $x_{0}$ is the distance between the secondary crack nucleus and the primary crack front. Since $n<1$ for an ellipse, $x_{0} /\left(1-n^{2}\right)>x_{0}$, so that the centre of the ellipse is beyond the origin of the secondary crack, away from the primary crack front. The secondary crack nucleus lies to the right of the centre of the ellipse in Fig. 9b, which suggests, perhaps curiously, that the local primary crack started from the right-hand side of the ellipse, rather than from the left-hand side where the specimen crack front lies.
As the crack propagated from the origin, it formed "Wallner" lines which are evident in Fig. 9a. Such lines are formed from the interaction of the main crack front and elastic waves reflected from the outer boundaries of the testpiece [6]. Following Smekal [7], a vector diagram for the crack speed in terms of the elastic stress wave velocity may be constructed from the trace of the Wallner lines. It was found that $|\mathbf{V}| \approx 0.3|\mathbf{c}|$, where $c$ is the sonic velocity, so with $c=1.5 \mathrm{~km} \mathrm{sec}^{-1}$ for PMMA at $193 \mathrm{~K}$, we deduce that the crack velocity in this particular case was $\sim 500 \mathrm{~m} \mathrm{sec}^{-1}$. We note that this is probably greater than the order of magnitude limiting velocity for quasi-static cracking given by Gurney and Ngan [8], beyond which kinetic effects must be considered in cracking analyses.

The fan-shaped smooth region at crack initiation in Fig. 9a shows a contrast with the adjacent rough regions. Shand [9] observed similar features on the fracture surface of a rectangular glass bar broken in bending, for which the following qualitative explanation was given. As shown in Fig. 10, the crack front at first advances radially making a half circle crack front. As the circular crack front increases its diameter, the effective stress intensity factor away from the centre line increases due to the redistribution of stress. This increase in stress intensity at both sides of the circular crack causes the crack speed in the front region to accelerate so that rough surfaces are produced. Since the crack speed at the sides of the circular crack front is faster than at the middle, the shape of the front changes from a circular arc to a flatter shape. The approximate dividing lines between

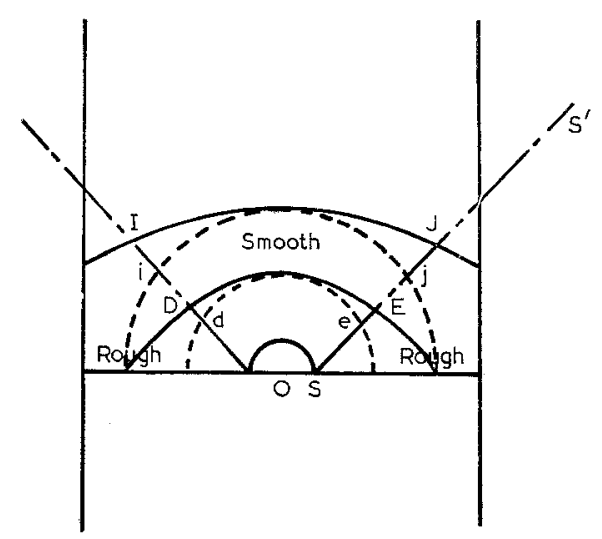

Figure 10 Qualitative explanation for fan-shaped smooth region and rough surface beyond in unstable fracture of Fig. 9 (see text for explanation). 


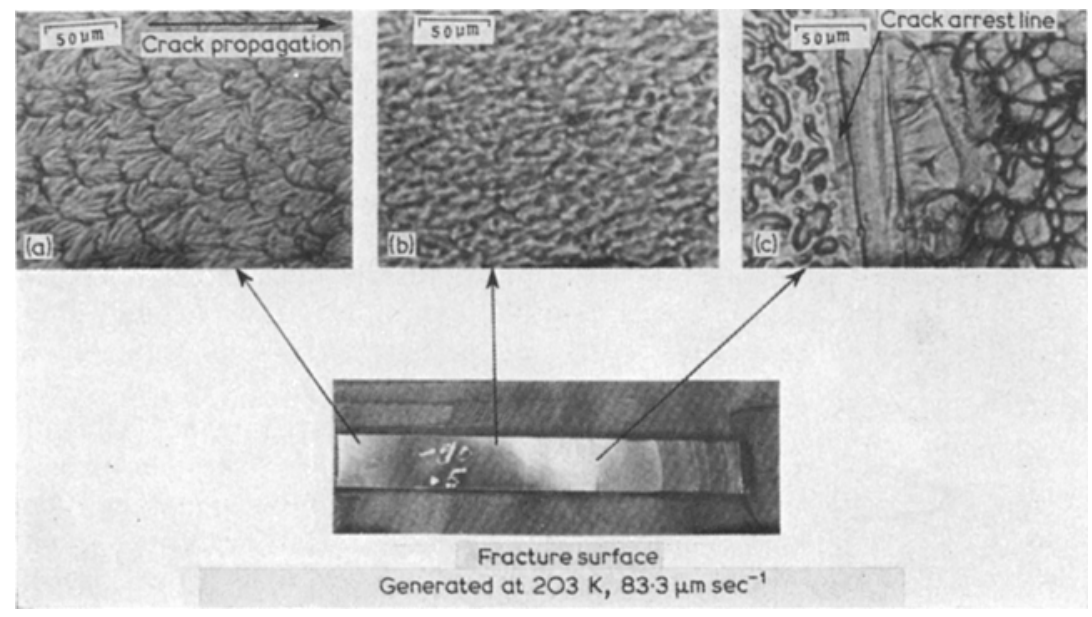

Figure 11 Changing features of unstable crack advancing in a jerky fashion. Before crack arrest, the initial conical markings change to granular. the low crack speeds which leave a smooth fracture surface and the high crack speeds which leave a rough fracture surface are shown on Fig. 10. As the crack propagates into the end region of the fracture specimen, its speed decreases and the fracture surface again becomes smooth.

In those experiments where crack arrest took place, the conical markings evident in the early part of the unstable propagation region gradually disappear as the crack advances to a point of arrest. Fig. 11 shows the changing features of the fracture surface as the unstable crack advances in a "jerky" manner. Markings initially conical gradually change to a more granular shape and the granular feature predominates up to the arrest point. If unstable cracking occurs after the arrest point, densely populated conical markings appear again. The granular feature appears consistently just ahead of every arrest point on most unstable fracture surfaces.

As reported by Cotterell [10], the density of the initial conical markings is dependent on the crack initiation toughness value, which varies
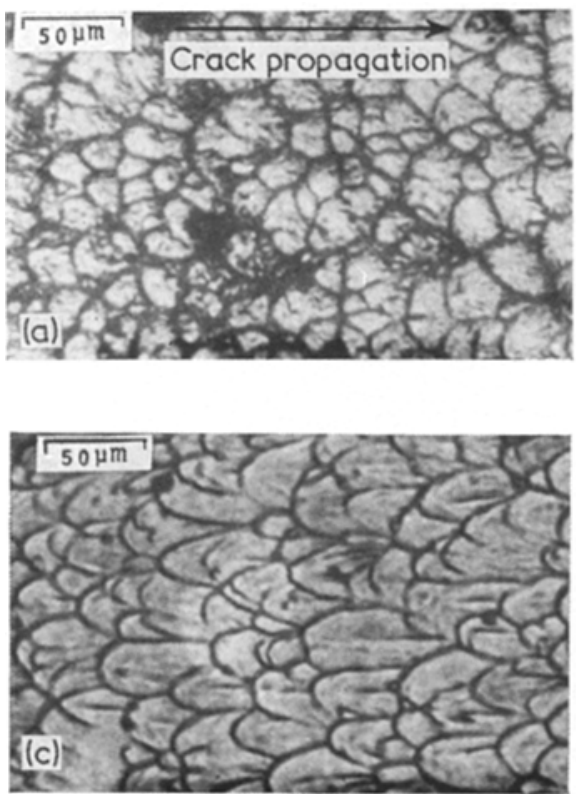
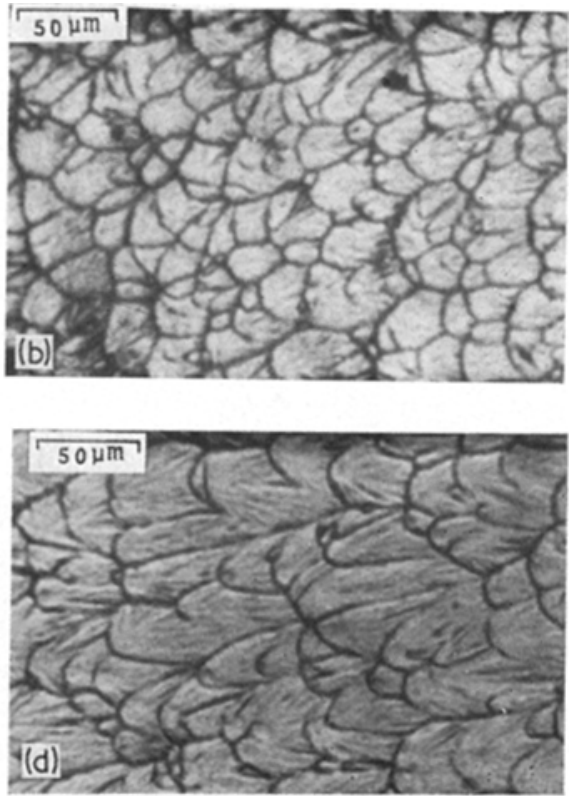

Figure 12 Variation in conical marking density at various values of the stress intensity factor. 
with temperature, cross-head speed and crack tip sharpness. Fig. 12 shows conical markings for unstably initiated cracks at different levels of toughness (given in terms of $K_{I C}$ in this instance); Fig. 8 is also relevant to this point.

\section{Transformations of fracture patterns}

A "toughness-biased" Ree-Eyring relationship described quite well the crack velocity for PMMA in the temperature range 283 to $353 \mathrm{~K}$, namely

$$
\dot{a}=A_{1} \exp \frac{-(U-\lambda R)}{k T}
$$

where $R$ is the fracture toughness determined by Gurney's sector area method, $U$ is the activation energy, $k$ is Boltzmann's constant, $T$ the absolute temperature, and $A_{1}$ and $\lambda$ are constants. A discussion of this equation and its relationship to the work of others is given by Atkins et al. [1]. The activation energy obtained in the range 283 to $353 \mathrm{~K}$ was some $86 \mathrm{~kJ} \mathrm{~mol}^{-1}$, which suggests secondary $\beta$-processes controlling crack propagation. This agrees with Boyer's contention [11] that crack growth is craze-controlled in glassy polymers. Fig. 13 shows the $R / T$ versus $\dot{a}$ relationships obtained in the range 243 to $353 \mathrm{~K}$; below $243 \mathrm{~K}$ no stable cracking was possible in the edge crack testpiece even at the lowest cross-head speed $(833 \mathrm{~nm} \mathrm{sec}-1)$. The right-hand side of the diagram, at the higher temperatures, is the region where Equation 1 was followed, (not including, of course, the unstable data points). Below $283 \mathrm{~K}$ transitions in toughness data are observed, with local peaks in toughness at the lower temperatures; these are discussed in [1].

Five different types of fracture surface

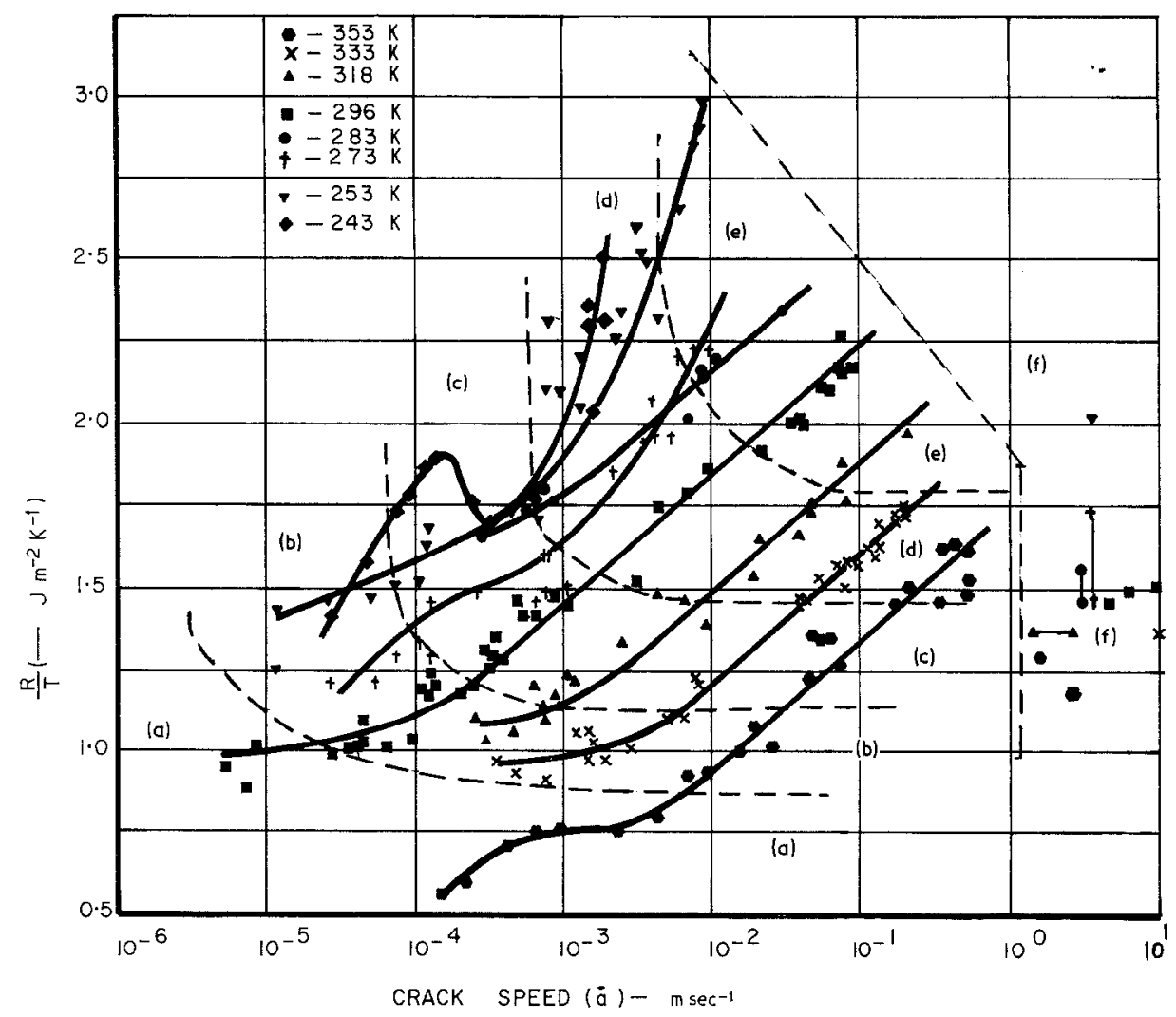

Figure 13 Semi-logarithmic plot of $R / T$ versus $\dot{a}$, at various temperatures, and associated fracture markings (see next page). Six arbitrary surface appearances have been identified. In the range where a linear relationship between $R / T$ and $\dot{a}$ is followed (i.e. a toughness-biased Ree-Eyring relationship) the fracture patterns may be shifted horizontally, (to a first approximation) analogous to visco-elastic transformations. 
A. G. ATKINS, C. S. LEE, R. M. CADDELL
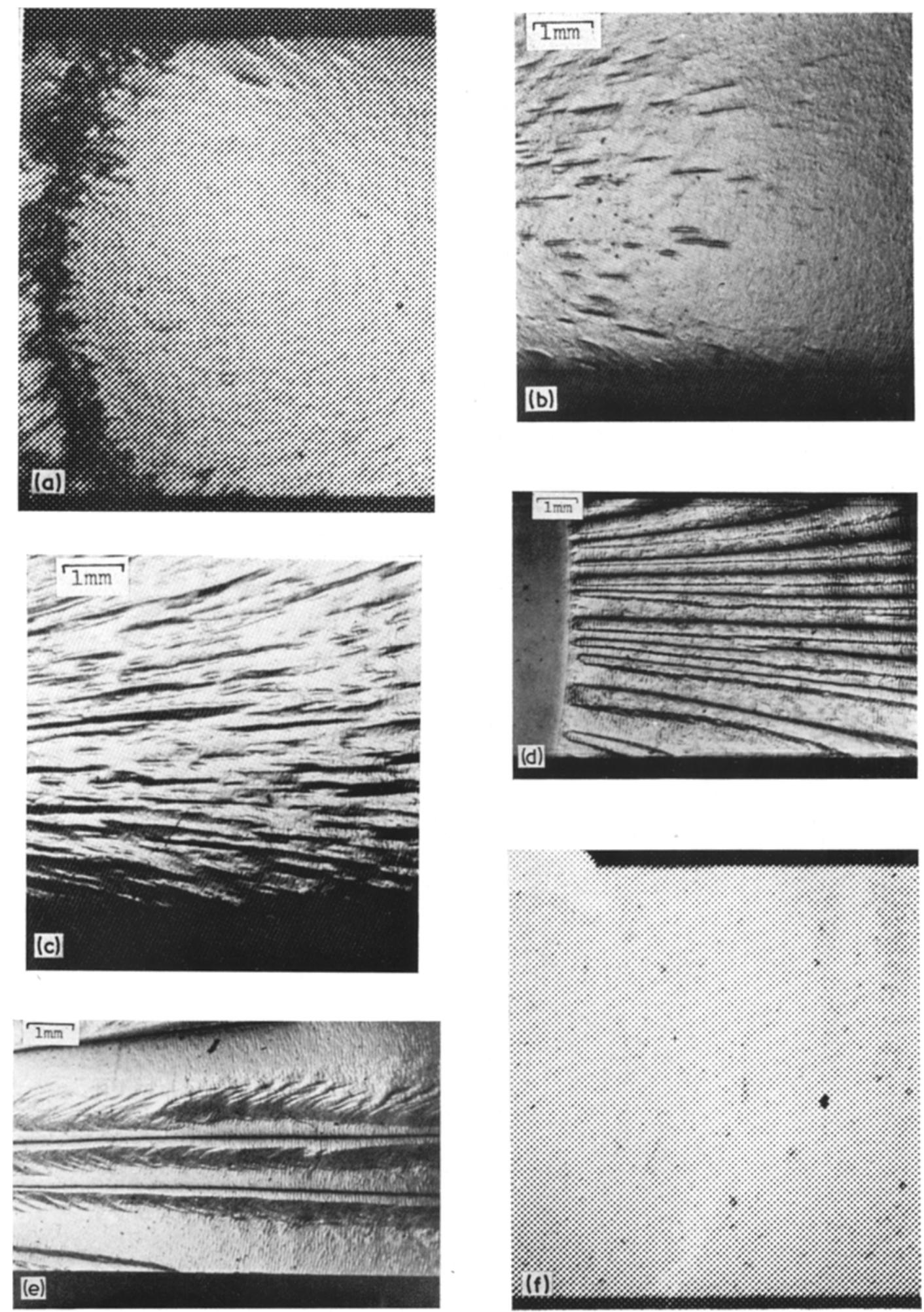

Fig. 13 
markings were arbitrarily identified as shown in Fig. 13. Transitions between the different types are gradual, but nevertheless approximate border lines have been superimposed on Fig. 13. In general terms, it seems that fracture patterns may be horizontally shifted to master $(R / T, \dot{a})$ combination, particularly in the region where the toughness-biased Ree-Eyring equation holds. Since no stable fracture is obtainable at temperatures below $243 \mathrm{~K}$ with this type of specimen, it is presumed that the type- $F$ fracture surface "swings over the top of the diagram". Perhaps the other border lines meet somewhere in the region of $\left\{R / T \approx 2\right.$ to $2 \frac{1}{2}, \dot{a}=10^{-4} \mathrm{~m}$ $\left.\mathrm{sec}^{-1}\right)$; this is conjecture on our part.

\section{Discussion and conclusions}

The fracture patterns provide a qualitative explanation for the molecular processes occurring in the fracture of PMMA. It appears from the observation of conical markings that unstable fracture in our low speed experiments is associated with the onset of secondary cracks ahead of the main crack front. Secondary cracks seem to start only when the fracture toughness reaches a threshold level of 600 to $700 \mathrm{~J} \mathrm{~m}^{-2}$, i.e. $R / T \approx 2$ to $3 \mathrm{~J} \mathrm{~m}^{-2} \mathrm{~K}^{-1}$ [1]. Under these conditions, an isothermal to adiabatic transition at the crack tip produces local softening and reduction in toughness $[1,3,4]$, which causes the test specimen's natural geometrical stability to be overcome [12]. Upper bound estimates for $R$ in the unstable range gave values smaller than the last stable value. The rough surfaces of multiplane crazing observed by Broutman and Kobayashi [13], and Green and Pratt [14], in quasi-stable cracking at much higher velocities, were not seen by us at these lower velocities.

The fracture markings in stable crack growth regions seem to show the involvement of voids. Voids in the craze material coalesce as a function of the fracture toughness as discussed by Cotterell [10], and Lednicky and Pelzbauer [15]. In the low crack speed region, the associated fracture toughness is so small that only a few cavities are formed and they show up as dimple marks on the fracture surfaces (Fig. 8). As the crack speed increases, the fracture toughness increases and more voids coalesce, giving needle-like features. Further increase in crack. speed, with an increase in fracture toughness, promotes more coalescence of voids, thereby changing the needle-like features into coarser, rib-like markings. These coarse rib markings suddenly change to flat, featureless surfaces as the fracture mode changes from stable to unstable propagation.

In the unstable region, the change in conical marking densities can be explained as follows: since the crack speed increases with increasing toughness, only a limited time is available for the holes in the craze material to coalesce. Thus the total number of the effective secondary fracture nuclei increases with higher $K_{\mathrm{IC}}$ or $R$, producing a higher density of conical markings on the fracture surfaces.

A ramification of the Lednicky and Pelzbauer theory of secondary crack formation is that the cavity size at the foci of the conical markings will vary as a function of $K_{\mathrm{IC}}$ level (or crack speed), because the cavity diameter is determined by the number of voids successfully coalesced during the crack propagation. Measurement of cavity diameter as a function of toughness may provide a means of testing the validity of their explanation of secondary crack formation; however, it was not attempted in the present investigation.

Finally, observations of various fracture patterns produced in a film of petroleum jelly sandwiched between two transparent plates deserves comment. When such a sandwiched film is broken by parting the plates slowly, patterns very similar to the needle-like markings on the stable fracture surfaces of PMMA may be observed. However, if the plates are separated by impact loading, the film shows features akin to the densely populated conical markings characteristic of unstable PMMA fracture. Similar observations have been made by Hays and Feiten [16] who showed the evolution of cavity formation in a thin viscous film between two parallel plates as the plates were separated at high velocity. High speed photography showed that in the early stages only a few cavities form and they grow in a "fern-like" shape, which is similar to the acicular features on the PMMA fracture surfaces. As the separation of the two plates proceeded at high speed, more cavities were formed and they grew to meet each other, forming features similar to the conical markings on the fast, unstable PMMA fracture surfaces. Since craze material ahead of a crack tip can be considered as a viscous film with high viscosity, these observations might lead to a method of modelling low speed fracture processes in glassy polymers. 


\section{References}

1. A. G. ATKINS, C. S. LEE and R. M. CADDELL, $J$. Mater. Sci. 10 (1975) 1381.

2. C. GURney and J. hunt, Proc. Roy. Soc. (Lond.) A299 (1967) 508.

3. R, P. KAMBOUR and R, E. BAR Ker, J. Polymer Sci. A2 4 (1966) 359.

4. J. G. Williams and G. P. Marshall, Polymer Letters 15 (1974) 251.

5. E. H. ANDREWS, in "Fracture in Polymers" (American Elsevier, New York, 1968).

6. H. Wallner, Z. Physik 114 (1939) 368.

7. A. SMEK AL, Glastech. Ber. 23 (2) (1950) 57.

8. C. GUR NEY and K. M. NGa , Proc. Roy. Soc. (Lond.) A325 (1971) 207.

9. E. B. ShAND, J. Amer. Ceram. Soc. 37 (1954) 563
10. B. COTterelL, Int. J. Fract. Mech. 4 (1968) 209.

11. R. F. BOYER, Polymer Eng. Sci. 8 (1968) 161.

12. C. GURNEY and Y. W. MAI, Eng. Fract. Mech. 4 (1972) 853.

13. L. J. broutman and T. Kobayashi, Proceedings of the International Conference on Dynamic Crack Propagation (Lehigh University), edited by $\mathrm{G}$. Sih (Noordhoff, Groningen, 1973) p. 215.

14. A. K. GREEN and P. L. PRATT, Eng. Fract. Mech. 6 (1974) 71.

15. F. LEDNICKY and Z. PELzBAUER, J. Polymer Sci. C 38 (1972) 375.

16. D. HAYS and J. FEITEN, in "Cavitation in Real Liquids", edited by A. B. Davis (American Elsevier, New York, 1964).

Received 21 October 1974 and accepted 31 January 1975. 